\title{
Nucleus of the active Centaur C/2011 P2 (PANSTARRS) ${ }^{\star}$
}

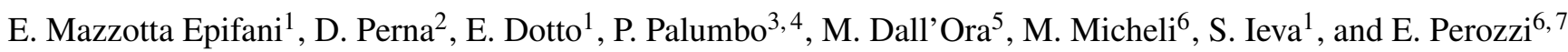 \\ 1 INAF-Osservatorio Astronomico di Roma, via Frascati 78, 00040 Monte Porzio Catone, Roma, Italy \\ e-mail: elena.epifani@oa-roma.inaf.it \\ 2 LESIA, Observatoire de Paris, PSL Research University, CNRS, Sorbonne Universités, UPMC Univ. Paris 06, Univ. Paris Diderot, \\ Sorbonne Paris Cité, 5 place J. Janssen, 92195 Meudon, France \\ 3 Universitá Parthenope, Dipartimento di Scienze e Tecnologie, Centro Direzionale Isola C4, 80143 Napoli, Italy \\ 4 INAF-IAPS, via Fosso del Cavaliere 100, 00133 Roma, Italy \\ 5 INAF-Osservatorio Astronomico di Capodimonte, via Moiariello 16, 80131 Napoli, Italy \\ ${ }^{6}$ ESA SSA-NEO Coordination Centre, Frascati, Roma, Italy \\ 7 Deimos Space, Strada Buchesti 75-77, Bucharest, Romania
}

Received 29 February 2016 / Accepted 8 September 2016

\begin{abstract}
Aims. In this paper we present observations of the active Centaur C/2011 P2 (PANSTARRS), showing a compact comet-like coma at the heliocentric distance of $r_{\mathrm{h}}=9 \mathrm{au}$. The observations were obtained in the framework of a wider program on Centaurs aimed at searching for comet-like activity in several targets outside Jupiter's aphelion.

Methods. We analysed visible images of the Centaur taken at the TNG telescope in the $R$ filter to investigate the level of coma contributing to the target brightness and to derive information on its nucleus size.

Results. Centaur C/2011 P2 (PANSTARRS) shows a faint but still detectable comet-like activity, which accounts for more than 50\% to the observed brightness. The coma contribution has been subtracted in order to derive an estimate for the Centaur's diameter of $D \sim 16 \mathrm{~km}$, assuming an albedo of $A=0.07$ (average of albedo measured within the Centaur group). The results for Centaur C/2011 P2 (PANSTARRS) fit in the general picture of the group: Centaurs with smaller perihelion distance $q$ and semi-major axis $a$ are smaller than those remaining farther from the Sun during their orbital path, thus reinforcing the idea that active Centaurs are "comets in fieri".
\end{abstract}

Key words. minor planets, asteroids: general - minor planets, asteroids: individual: C/2011 P2

\section{Introduction}

Centaurs are minor bodies residing in the region of the solar system between the orbits of Jupiter and Neptune. They move on highly chaotic and unstable orbits, have orbital lifetimes of less than $10^{7} \mathrm{yr}$ (Holman \& Wisdom 1993), and are therefore considered brief residents of the gas giant planet region. As of July 2016, 290 objects $^{1}$ have been classified as pertaining to the Centaur group.

Centaurs are regarded as "transition objects" from the group of inactive Kuiper Belt objects (KBOs) outside Neptune's orbit to the group of the Jupiter family comets (JFCs) with aphelion close to Jupiter's semi-major axis. The prototype of these quintessentially transitional bodies is 2060 Chiron, which was discovered in 1977 (Kowal et al. 1977) and first classified as an asteroid. It was the first object in this new class for which the presence of a diffuse and persistent coma was reported (Meech \& Belton 1989; Luu \& Jewitt 1990). Owing to its brightness $\left(m_{R} \sim\right.$ 16 beyond $10 \mathrm{au}$ ), Chiron has been extensively studied as a case study for this peculiar class (e.g. Ortiz et al. 2015; Belskaya et al. 2010; Groussin et al. 2004; Romon-Martin et al. 2003;

* Based on observations collected at the Telescopio Nazionale Galileo (TNG), operated on the island of La Palma by the Centro Galileo Galilei of the INAF (Istituto Nazionale di Astrofisica) at the Spanish Observatorio del Roque de los Muchachos of the Instituto de Astrofísica de Canarias.

1 http://www. johnstonsarchive.net/astro/tnoslist.html
Fernandez et al. 2002; Meech et al. 1997; Bus et al. 1996). It has been clearly established that Chiron's surface is quite homogeneous and has neutral colours. Its spectrum and albedo are compatible with the presence of a mixture of water ice and refractory grains, and its variations in the amplitude of the light curve has been related with its variable cometary activity level. Very recently, the hypothesis of the existence of ring material around Chiron has been investigated in order to explain the variability of its water band during the long-term monitoring of the past decades.

Recent results (Bauer et al. 2013) indicate that a cometary nature could be considered for many objects in this group, meaning that many Centaurs can be considered as "comets in disguise", i.e. they may have been comets in the past and/or may become comets in the future. Actually, a small percentage of the members in the group $^{2}$ ( 28 as of July 2016 , i.e. $\sim 10 \%$ of the total) have been observed with a diffuse coma and/or a tail in optical images.

Not many active Centaurs have been studied in detail, but the analysis of their comet-like environment (in a few cases performed by means of long-term monitoring along the target's orbital branch) shows a variety among the behaviour of the objects and demonstrates a compelling interest in the field. Mazzotta Epifani et al. (2006, 2011) investigated the cometary dust environment of P/2004 A1 (LONEOS), showing that this

\footnotetext{
2 https://physics.ucf.edu/ yfernandez/cometlist.html
} 
Centaur has a dust production rate around $100-200 \mathrm{~kg} / \mathrm{s}$ up to $6.5 \mathrm{au}$, comparable to several active comets at much shorter heliocentric distances. Conversely, the Centaur-comet transition object P/2010 C1 (Scotti) (Mazzotta Epifani et al. 2014a) has been identified as a rather small (radius $<4.8 \mathrm{~km}$ ), weak dust emitter $(0.1-15 \mathrm{~kg} / \mathrm{s}$ at $\sim 5.5 \mathrm{au})$. The unusual active Centaur $\mathrm{P} / 2011$ S1 (Gibbs) (Lin et al. 2014) has been identified instead as a small (radius $<4 \mathrm{~km}$ ) but very active dust emitter $(\mathrm{d} M / \mathrm{d} t \sim 100 \mathrm{~kg} / \mathrm{s})$, with a mass-loss rate per unit surface as high as that of the giant comet 29P/Schwassmann-Wachmann 1. The Centaur 166P/2001 T4 (NEAT) presented a variable activity level around a heliocentric distance of $r \sim 8.5$ au (Bauer et al 2003) and was observed still active at $r=12$ au post- $q$ (Shi \& Ma 2015). An upper limit of the nucleus radius of $a_{N}<14 \mathrm{~km}$ has been derived, with a rather intense dust production rate (at this heliocentric distance) of $252 \mathrm{~kg} / \mathrm{s}$. Shi \& Ma (2015) indicated that crystallisation of the amorphous water ice is a possible driving mechanism for such distant activity. The peculiar case of Centaur 174P/Echeclus (Bauer et al. 2008) is emblematic of the transitional nature of this class: previously characterised without a coma down to the $27 \mathrm{mag} \operatorname{arcsec}^{-2}$ level for more than $3 \mathrm{yr}$ (Rousselot et al. 2005), it was observed in strong outburst on 30 December 2005 (Choi et al. 2006), when it was at $r \sim 13 \mathrm{au}$. The comet-like activity was intense (dust production range estimates ranging from $1.7-4 \times 10^{2} \mathrm{~kg} / \mathrm{s}$ ) and with a peculiar coma morphology: the coma central condensation was in fact clearly separated by the Echeclus nucleus in a coma structure stable over several days (Bauer et al. 2008). The extreme case of 167P/CINEOS, which has the largest perihelion distance ( $q=11.8 \mathrm{au}$ ) among the active Centaurs, has been used as a case study for numerical simulations of the survival of amorphous water ice against crystallisation, which is considered the most probable trigger of comet-like activity at such heliocentric distances (Guilbert-Lepoutre 2012). Jewitt (2015) has recently shown, based on a sample of 37 targets (12 of which with evidence of activity), that active Centaurs tend to be those with smaller perihelion distances, as is expected for comet-like activity driven by a thermal process.

This growing interest in Centaurs, and in particular in the subgroup of active Centaurs, is driven by their link with other groups of minor bodies in the solar system (KBOs, JFCs): assessing their genetic and/or dynamic relationship is a major issue in order to constrain the past and current evolution of minor bodies in the system.

This paper reports on the observations of cometary Centaur C/2011 P2 (PANSTARSS) (Wainscoat et al. 2011), hereafter called P2 for clarity. We targeted P2 among the general framework of a wider program on Centaurs aimed at searching for comet-like activity in several targets outside Jupiter's aphelion. Several targets $(>20)$ have already been observed with mediumsized telescopes (TNG, CAHA, NOT) in order to homogeneously explore their surface colours. The observing program is currently in progress: the results will be used, together with the existing data in the literature, to clarify the real fraction of active Centaurs in the group and to statistically extend the analysis of the typical bimodal colour-colour distribution of the Centaur population.

P2 was discovered in August 2011 on CCD images taken with the "Pan-STARRS 1" $1.8 \mathrm{~m}$ Ritchey-Chretien telescope at Haleakala (Hawaii) when it was at heliocentric distance $r=$ $6.3 \mathrm{au}$ (outbound) and at geocentric distance $\Delta=5.3 \mathrm{au}$. Its comet-like nature was recognised thanks to the distinct nonstellar profile of the object, subsequently confirmed by visible
Table 1. Present orbital parameters of active Centaur P2.

\begin{tabular}{cccccc}
\hline \hline$e^{a}$ & $a^{b}[\mathrm{au}]$ & $q^{c}[\mathrm{au}]$ & $i^{d}\left[{ }^{\circ}\right]$ & $P^{e}[\mathrm{yr}]$ & $T_{J}^{f}$ \\
\hline 0.370 & 9.76 & 6.15 & 8.99 & 30.5 & 3.047 \\
\hline
\end{tabular}

Notes. ${ }^{(a)}$ Eccentricity; ${ }^{(b)}$ semi-major axis; ${ }^{(c)}$ perihelion distance; ${ }^{(d)}$ inclination; ${ }^{(e)}$ period; ${ }^{(f)}$ Tisserand parameter.

images taken by M. Micheli and G. T. Elliott a few days after its discovery with the $2.24 \mathrm{~m}$ University of Hawaii reflector.

The present orbital parameters of $\mathrm{P} 2$ are summarised in Table 1, as derived by the JPL Horizon DataBase ${ }^{3}$. The current orbit of P2 is dominated by its interaction with Saturn, the only major planet in its neighbourhood. However, owing to the significant eccentricity $(e \sim 0.37)$ and moderate inclination $\left(i \sim 10^{\circ}\right)$ of its orbit, the MOID (Minimum Orbital Intersection Distance) of P2 with Saturn is currently 0.33 au, and has been larger than $0.2 \mathrm{au}$ for at least the past four centuries. As a result, the orbit of the object has been moderately stable for the last few centuries, and for that period the object never came closer than $\sim 5.5$ au to the Sun.

A slightly smaller perihelion distance, and a consequently higher chance of close approaches with Jupiter, may have been possible in the past and could have been responsible for a more dramatic evolution of the object's orbit. An extrapolation beyond two millennia in the past is therefore difficult because of the chaotic nature of this region of phase space in the solar system together with the unknown non-gravitational behaviour of the object.

The purpose of this paper is to report on the persistent cometlike activity of P2 $4 \mathrm{yr}$ after its discovery, and to investigate in greater detail the properties of its nucleus (mainly the size).

Section 2 is devoted to the description of the observations and of the data reduction. In Sect. 3 we describe the observational results and discuss the derived analysis of the nucleus size of $\mathrm{P} 2$, comparing it with other active Centaurs in the same subgroup.

\section{Observations and data reduction}

P2 was observed on 2 August 2015 with the 3.58 m Telescopio Nazionale Galileo (TNG) at the Observatorio del Roque de los Muchachos (La Palma, Canary Islands). TNG images were obtained with the Device Optimised for the LOw RESolution (DOLORES) instrument, a focal reducer instrument installed at the Nasmyth B focus of the TNG. The detector is a $2048 \times 2048$ E2V 4240 thinned back-illuminated, deep-depleted, Astro-BB coated CCD with a pixel size of $13.5 \mu \mathrm{m}$. The scale is 0.252 arc$\mathrm{sec} / \mathrm{px}$, which yields a field of view of about $8.6 \times 8.6 \mathrm{arcmin}^{2}$. The instrument was equipped with the broadband filters $B, V, R$, and $I$ of the Johnson-Cousins system. Unfortunately, the conditions of the observing night were not optimal (2 days from the full moon), the sky was very luminous, and we were only able to obtain reliable data for the target in the $R$ filter. Favourably, the seeing was good and stable during the observations (the instrumental ORM DIMM seeing was measured $\sim 0.5 \operatorname{arcsec}$ at the telescope).

The geometry of observations of $\mathrm{P} 2$ are summarised in Table 2.

Even if P2 was quite far from the Sun (see Table 2) and therefore rather slow against the star background, it was followed with

http://ssd.jpl.nasa.gov/horizons.cgi 


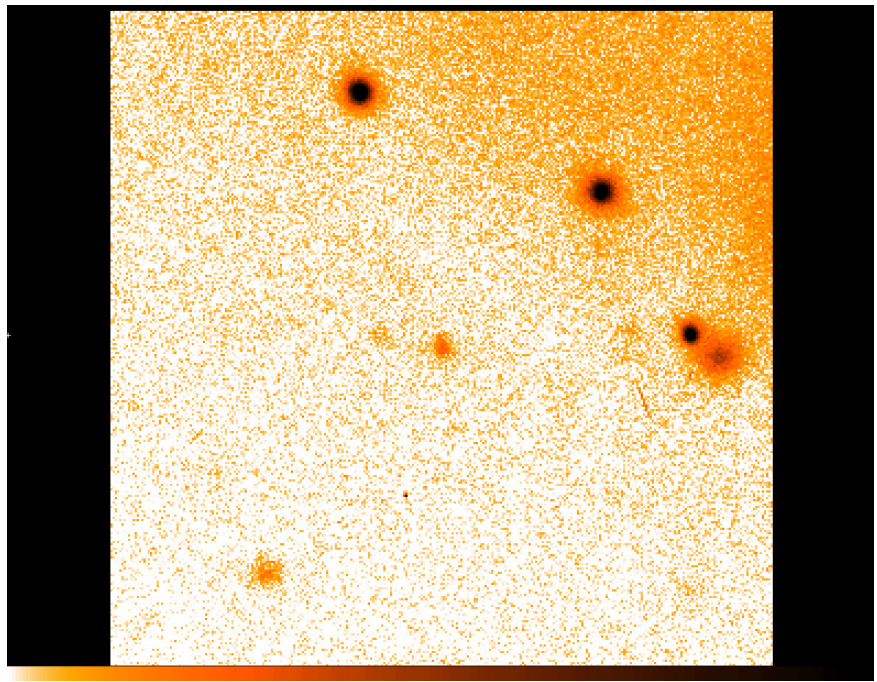

Fig. 1. Final image of $\mathrm{P} 2$ in the $R$ filter, obtained as coadding of four individual frames taken consecutively in the time range 03:11-03:32 UT displayed in log scale to enhance the low-level activity. The median air mass is 1.394 . The size of the image is $4.8 \times 10^{5} \mathrm{~km}$. North is up and east is to the left.

the telescope tracking at the non-sidereal rate and a sequence of 16 images was acquired (exposure time of $300 \mathrm{~s}$ each) for a total of $\sim 1.5 \mathrm{~h}$ of observations in the four BVRI broadband filters. Unfortunately, as said before, only the images acquired in the $R$ filter are useful for a reliable analysis. The $R$-filter sequence (four consecutive images) started at 03:11 UT when the target was at an angular distance $\theta=39.9^{\circ}$ from the moon (illuminated fraction 90\%). In each frame, P2 appeared as a stellar object, moving very slowly against the star background.

All the images were reduced and calibrated using standard procedures (subtraction of master bias and flat-field correction) with the aid of the ESO Munich Image Data Analysis System (MIDAS 1998) software package. The absolute calibration of the magnitudes was obtained by computing the photometric zero points and extinction coefficients by a least-square fit for several Landolt (1992) standard fields.

To increase the signal-to-noise ratio $(\mathrm{S} / \mathrm{N})$ for the image analysis, we decided to co-add the four frames obtained in the $R$ filter: the position of the P2 optocentre in each image was determined by fitting a two-dimensional Gaussian to the innermost pixels of the coma. The images were then recentred using this optocentre and summed. Sky correction was performed by subtracting a first-order polynomial sky approximation computed from the pixel areas containing no stars. The sky image was built for each frame via a standard MIDAS routine ${ }^{4}$.

The final $R$ image of $\mathrm{P} 2$ is shown in Fig. 1.

\section{Faint activity and nucleus size}

From Fig. 1, it is possible to see that P2, observed at a heliocentric distance to more than 9 au, shows an amost stellar appearance.

Jewitt (1991) showed that a compact coma may be unresolved and yet still influence or even dominate the photometry of an intrinsically faint target. In order to verify whether the bare

\footnotetext{
4 The areas for background are chosen interactively via the cursor. Then, the routine creates the sky image from a 2D polynomial, using the least-square method.
}

Table 2. Geometry of observation of P2 at TNG.

\begin{tabular}{ccccc}
\hline \hline Date $^{a}$ & $r^{b}[\mathrm{au}]$ & $\Delta^{c}[\mathrm{au}]$ & $\mathrm{PA}^{d}\left[{ }^{\circ}\right]$ & $\alpha^{e}\left[{ }^{\circ}\right]$ \\
\hline 3 August 2015 02:11 UT & 9.03 & 8.75 & 248.8 & 6.3 \\
\hline
\end{tabular}

Notes. ${ }^{(a)}$ Beginning of the image sequence in the $R$ filter (see text); ${ }^{(b)}$ heliocentric distance; ${ }^{(c)}$ geocentric distance; ${ }^{(d)}$ position angle of the extended Sun-target radius vector as seen in the observer's plane of sky, measured counter-clock wise; ${ }^{e}$ phase angle.

nucleus or an unresolved coma was observed for P2, we adopted a technique which is often used to compare the target profile with a stellar one, obtained as the stellar point spread function (PSF) extracted by several standard frames obtained just before and after the target sequence. Some successful examples of the application of this technique, adopted to enhance even a weak dust coma in apparently stellar objects or to confirm the appearance of a bare nucleus, can be found in e.g. Licandro et al. (2000), Lowry \& Fitzsimmons (2005), Perna et al. (2013), and Mazzotta Epifani et al. (2008, 2014a).

Four profiles centred in the Centaur optocentre (in four different projected directions) were extracted and averaged to produce a mean target profile, and after normalisation to a value of 1 in the peak (the normalisation to 0 in the wings is ensured by the background subtraction), the profile was compared with the stellar one (derived by an average of several stars in several standard fields). The result is shown in Fig. 2 (left panel): the object is distinctly broader than the stars and there is a clear excess in its profile, confirming therefore that there actually is an unresolved coma and that P2 is active even at more than 9 au from the Sun.

Because the quality of the observing circumstances was not optimal, we also performed an analysis of the surface brightness profile (SBP) of P2, computed by azimuthally averaging the signal within concentric annuli around the optocentre. This is done in order to obtain the surface brightness (SB) $\Sigma_{\lambda}(\rho)$ (given in magnitude and computed for a unit solid angle $\left.\left[\operatorname{arcsec}^{2}\right]\right)$ at various aperture radii $\rho$ [arcsec]. The SBP of P2 is compared to the stellar SBP(s) in Fig. 2 (middle panel); the stellar SBPs reported in the figure were obtained as the average of 34 stars in eight star fields at four air mass values (also to give an idea of the quality of the night). The SBP of P2 is clearly higher than the average stellar profile, giving a clear indication of the presence of a poorly resolved coma around the Centaur's nucleus.

As an additional test, we analysed the distribution of the point spread function (PSF) profiles on the stacked image we used for our photometric measurements by means of the software SExtractor (Bertin \& Arnouts 1996). SExtractor is widely adopted in the literature to automatically distinguish point and extended sources, and to perform astrometric and photometric measurements. The advantages of running SExtractor on the stacked image is twofold: first, as said above, the faint active Centaur has a higher $\mathrm{S} / \mathrm{N}$ on the stacked image, and therefore the shape analysis has a more robust statistical basis; second, the PSF of the (standard) stars can vary from image to image because of the deformation effects on the main mirror at different pointings (due to gravity), because of possible significant seeing variations, and because of small inhomogeneities in the tracking. In particular, we adopted three shape classifiers, namely the CLASS_STAR (a neural network-based parameter, which attributes different numerical flags to sure stars, cosmic rays, and extended sources), the FLUX_RADIUS (the radius which contains half of the source flux), and the FWHM; all three classifiers are evaluated on each individual source. In Fig. 2 (right panel) 

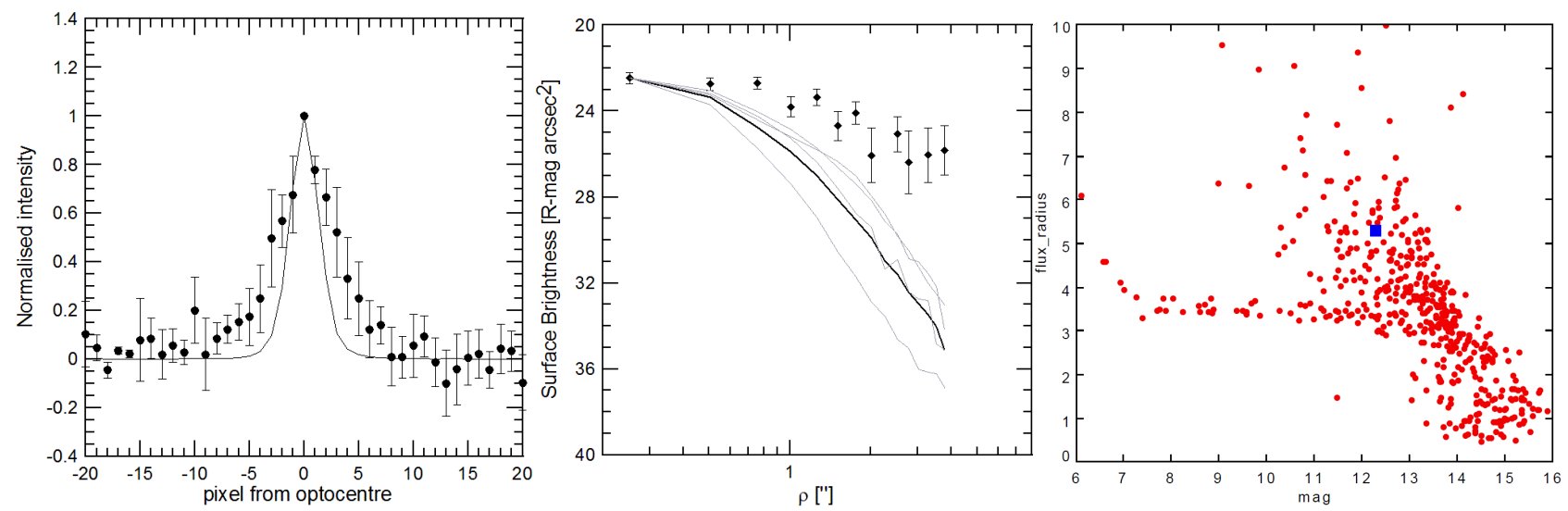

Fig. 2. Weak cometary activity of Centaur P2. (Left) Comparison of the averaged P2 profile (black points; error bars on points are due to the dispersion of values among the four extracted profiles) with the average stellar PSF profile (continuous line). (Middle) P2 SBP comparison with the stellar SBP, for four air mass values during the night (the thick line is the average value). (Right) Run of the SExtractor FLUX_RADIUS classifier (see text for details) as a function of the instrumental magnitude. The red dots represent the individual sources, while the blue square marks the position of P2. The straight horizontal sequence is formed by sources classified as sure stars. P2 is clearly above this sequence in the extended sources region.

we show the run of FLUX_RADIUS as a function of the (instrumental) magnitude. The locus of the stars is clearly defined as a straight horizontal sequence, while the position of $\mathrm{P} 2$ is in the region of extended sources, clearly separated from the sure stars sequence. The plots of the CLASS_STAR and of the FWHM as a function of the magnitude show similar results. Therefore, also on the basis of the SExtractor analysis, we can confirm that P2 is a definitely extended source.

In order to quantify the coma contamination to the photometry of P2, we applied the same technique adopted in Mazzotta Epifani et al. $(2007,2008)$ to evaluate nuclear sizes of distant JFCs observed with apparent stellar appearance. Jewitt (1991) showed that for a standard steady-state coma, the integrated coma magnitude $m_{\text {coma }}$ within a diaphragm of radius $\rho$ is related to the SBP at $\rho$ as

$m_{\text {coma }}=\Sigma_{\lambda}(\rho)-2.5 \log \left(2 \pi \rho^{2}\right)$.

The approximation of a steady-state coma can be questioned, especially in the presence of a non-steady dust appearance such as jets, but in the case of a stellar appearance it can be used to give an estimate of the amount of coma contamination to the bare nucleus. This can be carried out by considering that

$\frac{F_{\text {coma }}}{F_{\text {TOT }}}=10^{-0.4\left(m_{\text {coma }}-m_{\text {ToT }}\right)}$.

To select the reference aperture, we used the "growth curve" technique and determined the aperture that included $\sim 90 \%$ of the photometric light (it was $\rho=1.51^{\prime \prime}$, corresponding to $1.1 \times 10^{4} \mathrm{~km}$ at the Centaur's distance). In this reference aperture, the integrated magnitude in the $R$ filter to be used in Eq. (2) was $m_{\mathrm{TOT}}=21.29 \pm 0.03$.

Using the above reference aperture $\rho$ in (1), this value allowed us to determine the reference integrated coma magnitude to substitute in Eq. (2), and to finally obtain the level of coma contamination for P2. The observations of August 2015 resulted in $\frac{F_{\text {coma }}}{F_{\mathrm{TOT}}}=0.62$, i.e. the stellar appearance of $\mathrm{P} 2$ can likely be considered largely contaminated by the presence of a compact, unresolved coma around its nucleus. Then, considering that $\frac{F_{\text {nucleus }}}{F_{\text {TOT }}}=1-\frac{F_{\text {coma }}}{F_{\text {TOT }}}$ and that an expression similar to (2) can also be written for $\frac{F_{\text {nucleus }}}{F_{\text {TOT }}}$, the apparent nuclear magnitude $m_{\text {nucleus }}$ can be easily obtained as

$m_{\text {nucleus }}=m_{\mathrm{TOT}}-2.5 \log \left(1-\frac{F_{\text {coma }}}{F_{\mathrm{TOT}}}\right)$.

For P2, Eq. (3) gives $m_{\text {nucleus }}=22.35$ (in the $R$ filter).

The nucleus magnitude derived above can be used to compute the geometrical cross-section of the Centaur nucleus using the Russell (1916) expression, reformulated by Jewitt (1991), which in the case of a spherical object is given by

$A a^{2}=2.24 \times 10^{22} r^{2} \Delta^{2} 10^{\left(m_{\mathrm{SUN}}-m_{\text {nucleus }}+\alpha \beta\right)}$,

where $A$ is the geometric albedo, $a[\mathrm{~m}]$ is the radius of the body, $m_{\text {SUN }}$ the magnitude of the Sun in the same wavelength band of the body $\left(m_{\mathrm{SUN}}=-27.09\right.$, see Holmberg et al. 2006), $r$ and $\Delta$ are respectively the heliocentric and geocentric distance of the target, and $\alpha$ and $\beta$ are respectively the phase angle $\left[{ }^{\circ}\right]$ and the phase coefficient $\left[\mathrm{mag} /{ }^{\circ}\right]$ in the phase-darkening function $\Phi(\alpha)=10^{-0.4 \alpha \beta}$.

The values of many of the above parameters are not known for P2 (as for the majority of the minor bodies orbiting in our solar system); nevertheless, some realistic assumptions can be made and a reliable value for the Centaur's nucleus can be derived based on what we know for similar targets from the most recent observations.

In particular, according to Peixinho et al. (2004), who derived it from a least-square fit of the linear $\Phi(\alpha)$ to the published data for the Centaur group, we can assume $\beta=0.11 \mathrm{mag} /{ }^{\circ}$. This value is very similar to the value $\beta=0.1$ used by Jewitt (2009) in his survey on Centaurs, and is the same used in other portraits of single active targets, e.g. P/2010 C1 (Scotti; Mazzotta Epifani et al. 2014a), allowing therefore a more reliable comparison of derived nucleus size values.

As far as albedo is concerned, a wide spread of values has been measured within the Centaur group: from $2.5 \%$ to $26 \%$, with a mean value of $6.9 \% \pm 3.9 \%$ (Duffard et al. 2014). Therefore, by means of Eq. (4) we decided to produce a "diameter curve" to obtain information on the nucleus size of $\mathrm{P} 2$, as shown in Fig. 3. For the mean albedo, we obtain $D=16 \pm 8 \mathrm{~km}$ (the 


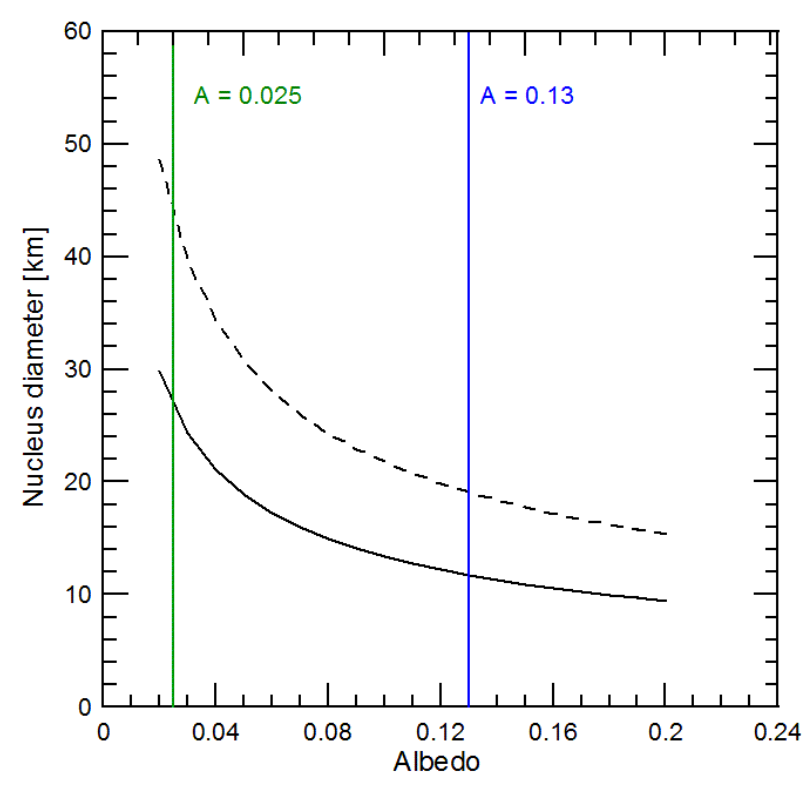

Fig. 3. "Diameter curve" for the active Centaur P2. The continuous curve corresponds to nucleus diameter obtained with the nucleus magnitude $m_{\text {nucleus }}$ (after the subtraction of coma contamination). The dashed curve is the "upper limit" on the nucleus size, obtained with the total

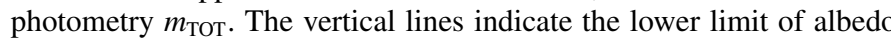
values derived in the class (green line, Duffard et al. 2014) and the value measured for the two "canonical" active Centaurs (blue line) Chiron (Bus et al. 1989) and 29P/Schwassmann-Wachmann (Cruikshank \& Brown 1983).

corresponding value of the upper limit, which we would assume if we did not apply the "coma subtraction" technique described above, is $26 \mathrm{~km}$ ).

The SBP has also been used to derive a lower limit for the Af $\rho$ value (A'Hearn et al. 1984), which is often considered as a proxy for the dust production. Following Eq. (1), the coma magnitude in the reference aperture of 1.51 arcsec (corresponding to $\rho=10^{4} \mathrm{~km}$ ) was extracted, and an A $\rho=161 \pm 4 \mathrm{~cm}$ was derived. If compared with values obtained at such large heliocentric distances for other active bodies (see e.g. Fig. 5 in Mazzotta Epifani et al. 2014b), it can be seen that P2 is quite active among the Centaur class, even if the statistics are still too poor to derive significant conclusions. To derive a more quantitative estimate of the dust production rate, we decided to apply two independent techniques which were successfully used in the past. It should be borne in mind, however, that both the techniques are based on a number of assumptions about the comet-like dust environment (grain size and volumetric density, grain ejection velocity, and so on), and are then strongly model dependent.

The photometric method, derived from the method used by Jewitt (2009) to compute the dust production rate of active Centaurs in the region between 5 and 12 au, has been adapted and already successfully applied by our group to several active minor bodies such as long-period comets (Mazzotta Epifani et al. 2010, 2014b), short-period comets (Mazzotta Epifani \& Palumbo 2011), and active Centaurs (Mazzotta Epifani et al. 2011, 2014a). The model relates the optical photometry to the dust production rate via some realistic assumptions on the comet-like dust environment, in particular on the power-law dust size distribution and the dust outflow velocity. Details of the application of the model applied to the active Centaur P/2010 C1 (Scotti) can be found in e.g. Mazzotta Epifani et al. (2014a); here we report that for $\mathrm{P} 2$, adopting a power-law dust size distribution with power $q=-3.5$ for compact (bulk density $\delta=1000 \mathrm{~kg} / \mathrm{m}^{3}$ ) grains between $a_{-}=10 \mu \mathrm{m}$ and $a_{+}=1 \mathrm{~mm}$ and a mean dust outflow velocity of $v=5 \mathrm{~m} / \mathrm{s}$, the coma photometry among the coma radius of $\phi^{\prime}=1.51^{\prime \prime}$ and $\phi^{\prime \prime}=2.02^{\prime \prime}$ results in a dust production rate of $Q_{\mathrm{d}} \sim 4 \mathrm{~kg} / \mathrm{s}$.

On the other hand, using a modification of the technique described in detail in Meech \& Weaver (1996), Bauer et al. (2003) investigated the comet-like environment of the active Centaur 166P/NEAT (2001 T4) and derived its dust production rate as

$Q_{\mathrm{d}}=A f \rho\left(4 / 3 \pi a^{3} v_{\mathrm{ej}} \sigma\right)\left(\pi a^{2} p\right)$,

where $a$ is the dust mean grain radius, $v_{\mathrm{ej}}$ is the grain ejection velocity, $\sigma$ is the grain volumetric density, and $p$ the dust grain albedo. Adopting $a=1 \mu \mathrm{m}, v_{\mathrm{ej}}=1 \mathrm{~m} / \mathrm{s}, \sigma=1 \mathrm{grain} / \mathrm{cm}^{3}$, and $p=0.04$, i.e. the same values used by Bauer et al. (2003) for the active Centaur 166P/NEAT (2001 T4) (only the grain velocity has been decreased since very slow grains have been modelled at large heliocentric distances; see e.g. Mazzotta Epifani et al. 2009), we derive a $Q_{\mathrm{d}} \sim 54 \mathrm{~kg} / \mathrm{s}$ for $\mathrm{P} 2$. This range of dust production rate $\left(Q_{\mathrm{d}}=4-54 \mathrm{~kg} / \mathrm{s}\right)$ should only be regarded as a weak lower-upper bound; it does not provide a strong constraint on the actual dust production rate for $\mathrm{P} 2$ since it depends on a number of unknown parameters of the dust. If we look at this range as the lower limit for the Centaur mass loss during an orbital revolution $(P=30.5 \mathrm{yr})$, assuming a radius of $8 \mathrm{~km}$ (see above) and a nucleus density of $500 \mathrm{~kg} / \mathrm{m}^{3}$, we could infer a weak lower limit on the erosion of a layer of $1-10 \mathrm{~cm}$ over the entire P2 surface during a single orbital period, compatible with the typical lifetime of a Centaur (Holman \& 1993).

\section{Discussion and conclusions}

Direct comparison among size measurements for Centaurs is rather difficult given the unknown parameters to adopt (albedo, phase coefficient) and the strict dependence of Centaurs' derived photometry on observing and analysis circumstances (e.g. stellar or extended appearance, reference aperture, possible coma subtraction). Nevertheless, we tried to summarise the current knowledge on the size of Centaurs in Fig. 4, where the diameter $D$ of 42 Centaurs is shown as function of the perihelion distance $q$, and in Fig. 5, where the diameter of 40 Centaurs is given as a function of the semi-major axis a (Jewitt 2009, 2015; Stansberry et al. 2008; Shi \& Ma 2015; Pál et al. 2015; Mazzotta Epifani 2011, 2014a; Lin et al. 2014; Duffard et al. 2014). For clarity, two values of $a$ outside the Centaur region (between Jupiter's and Neptune's orbits) have been removed from the sample (31 inactive targets and 11 active targets) in Fig. 5 ( $a=97$ au for 1999 TD10 and $a=829$ au for 2013 AZ60). The value presented for P2 in this paper is added to this general picture, taking into account some caveats for the interpretation of the results.

In our case, the derived value of $\mathrm{P} 2$ size can be more properly considered an upper bound on the nucleus size than an actual measurement since it is strongly dependant on the adopted values for unknown parameters (albedo, mainly). Also, using the photometry reported by Jewitt (2015) for P2 observed in October 2014, when the Centaur was at $r=8.39$ au $\left(m_{R}=22.04 \pm 0.05\right.$ in $\left.\rho=2.2 \times 10^{4} \mathrm{~km}\right)$, and adopting the same parameters used above ( $\beta=0.11 \mathrm{mag} /{ }^{\circ}$ and $\left.A=0.07\right)$, we can derive a different value for the upper limit of P2's size: $D \leq 18 \mathrm{~km}$ (the morphology of $\mathrm{P} 2$ is reported as "extended" by these authors, but no discussion is reported on the possible level of coma contamination). 


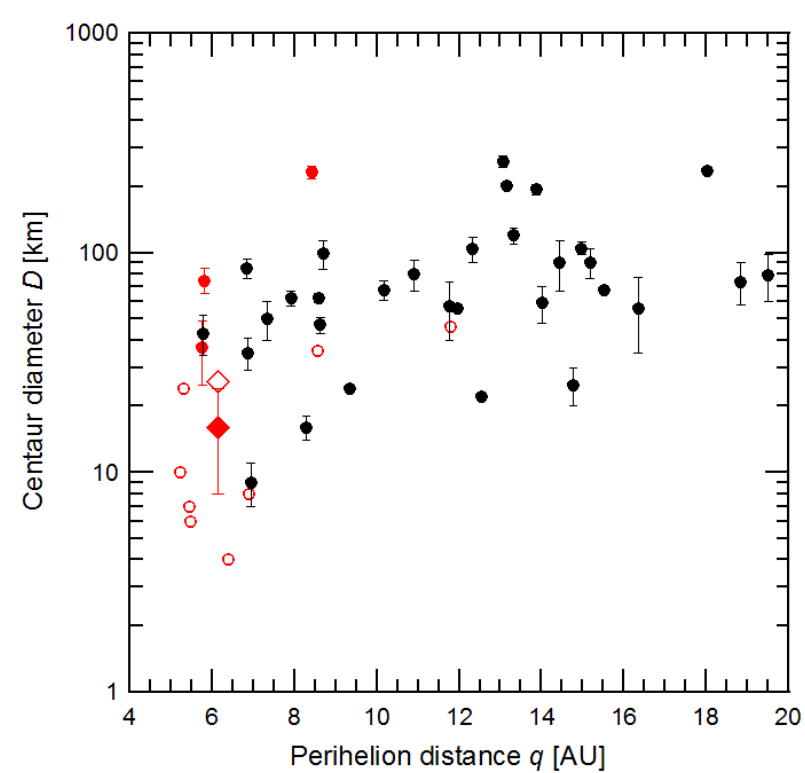

Fig. 4. Values (filled symbols) or upper limits (empty symbols) of diameter for inactive (black symbols) and active (red symbols) Centaurs, as per January 2016, plotted with respect to their perihelion distance $q$. Large red symbols are those derived for P2 in this paper for $A=0.07$ (filled diamond) and the upper limit without coma subtraction (empty diamond).

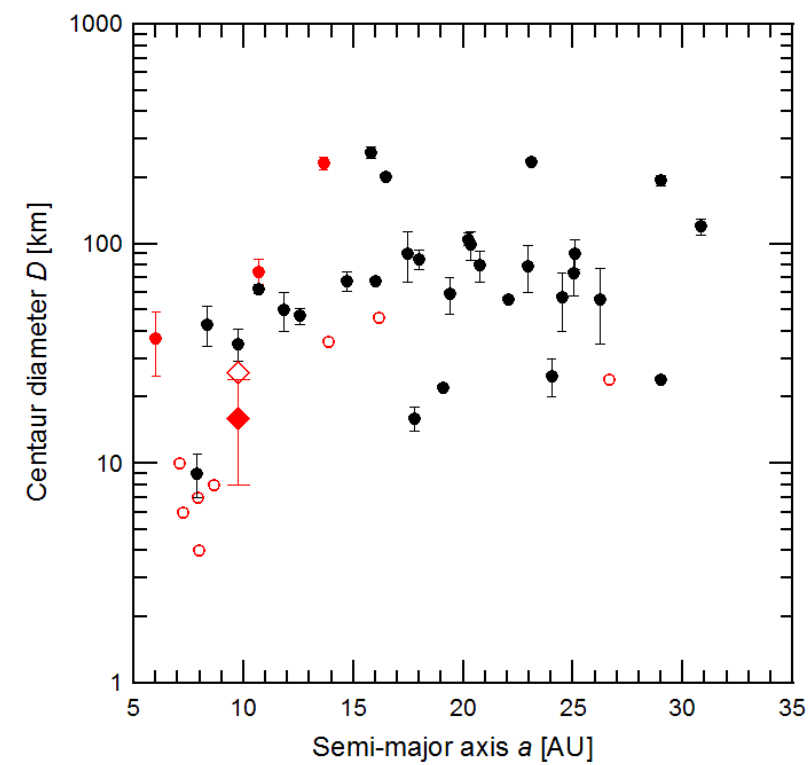

Fig. 5. Values (filled symbols) or upper limits (empty symbols) of diameter for inactive (black symbols) and active (red symbols) Centaurs, as per January 2016, plotted with respect to their semi-major axis $a$. Large red symbols are those derived for P2 in this paper for $A=0.07$ (filled diamond) and the upper limit without coma subtraction (empty diamond).

Moreover, here we present only a single photometry point, and it should not be forgotten that the albedo used in our analysis is actually the mid-point of two albedo clusters that correlate with object colours (see Tegler et al. 2008 and Bauer et al. 2013). The convolution of $\beta$ and $\alpha$ in Eq. (4), along with the uncertainty in $A$, make it difficult to utilise Eq. (4) meaningfully without multiple samplings at different phase angles and without rotational light curve information.
Finally, a few measurements are still available for a relatively small sample of Centaurs (less than 50 on a population of 290 members). Nevertheless, two general trends seem to emerge for the still immature general picture: Centaurs with smaller $q$ tend to be smaller than those which remain farther from the Sun during their orbital path, and active Centaurs tend to be smaller than inactive ones. The same picture seems to emerge when considering the $a$ of Centaurs (with the remarkable exception of the active Centaur C/2001 M10 (NEAT), which has the largest $a$ among the Centaurs with sustained activity).

Also, considering the large uncertainties of our analysis, the data obtained for P2 and presented in this paper fit into this general picture. It should be taken into account that both previous trends can be affected by non-negligible observing biases: small targets that are far away are difficult to observe without very large telescopes and long exposure times, and comet-like activity could be unresolved (and the target reported as inactive, with the appearance of a bare nucleus) when targets are observed at large heliocentric distances and an accurate comparison with a stellar profile is not performed. Jewitt (2009), on the basis of a sample of 23 targets, suggested that active Centaurs have smaller perihelia than the inactive ones (and also than all known Centaurs) because of a thermally driven comet-like activity. This would be also in agreement with the idea that active Centaurs are comets in fieri, reinforced also by the fact that the average short-period comet (whose group is considered to be continuously replenished by the Centaurs group) has a rather small average nucleus of $D=3.2 \mathrm{~km}$ (Lamy 2004).

More measurements are therefore needed on single targets pertaining to the group of Centaurs in order to carefully analyse the presence of comet-like activity (intermittent or sustained even if faint), and to assess their importance in constraining genetic and dynamical links within groups and families of small bodies orbiting in our system.

\section{References}

A'Hearn, M. F., Schleicher, D. G., Feldman, P. D., Millis, R. L., \& Thompson, D. T. 1984 , AJ, 89, 579

Bauer, J. M., Fernández, Y. R., \& Meech, K. J. 2003, PASP, 115, 981

Bauer, J. M., Choi, Y.-J., Weissman, P. R., et al. 2008, PASP, 120, 393 Bauer, J. M., Gray, T., Blauvelt, E., et al. 2013, ApJ, 773, 22

Belskaya, I. N., Bagnulo, S., Barucci, M. A., et al. 2010, Icarus, 210, 472 Bertin, E., \& Arnouts, S. 1996, A\&AS, 117, 393

Bus, S. J., Bowell, E., Harris, A. W., \& Hewitt, J. V. 1989, Icarus, 77, 223

Bus, S. J., Buie, M. W., Schleicher, D. G., et al. 1996, Icarus, 123, 478 Choi, Y.-J., Weissman, P. R., Chesley, S., et al. 2006, CBET, 563, 1 Cruikshank, D. P., \& Brown R. H. 1983, Icarus, 56, 377

Duffard, R., Pinilla-Alonso, N., Santos-Sanz, P., et al. 2014, A\&A, 564, A92

Fernandez, Y. R., Jewitt, D. C., \& Sheppard, S. S. 2002, AJ, 123, 1050

Groussin, O., Lamy, P., \& Jorda, L. 2004, A\&A, 413, 1163

Guilbert-Lepoutre, A. 2012, AJ, 144, 97

Holman, M. J., \& Wisdom, J. 1993, AJ, 105, 1987

Holmberg, J., Flynn, C., \& Portinari, L. 2006, MNRAS, 367, 449

Jewitt, D. 2009, AJ, 137, 4296

Jewitt, D. 2015, AJ 150, 201

Jewitt, D. C. 1991, in Comets in the Post-Halley Era, eds. R. L. Jr Newburn, M. Neugebauer, J. Rahe (Dordrecht: Kluwer), 1

Kowal, C. T., Liller, W., \& Chaisson, L. J. 1977, IAU Circ., 3147, 1

Lamy, P., Toth, I., Fernandez, Y. R., \& Weaver, H. A. 2004, in Comets II, eds. M. Festou, H. U. Keller, \& H. A., Weaver (The University of Arizona Press) Landolt, A. U. 1992, AJ, 104, 340

Licandro, J., Tancredi, G., Lindgren, M., et al. 2000, Icarus, 147, 161

Lin, H. W., Chen, Y. T., Lacerda, P., et al. 2014, AJ, 147, 114

Lowry, S. C., \& Fitzsimmons, A. 2005, MNRAS, 358, 641

Luu, J. X., \& Jewitt, D. C. 1990, AJ, 100, 913

Mazzotta Epifani, E., Palumbo, P., Capria, M. T., et al. 2006, A\&A, 460, 935 
E. Mazzotta Epifani et al.: The active Centaur C/2011 P2 (PANSTARRS)

Mazzotta Epifani, E., Palumbo, P., Capria, M. T., et al. 2007, MNRAS, 381, 713 Mazzotta Epifani, E., Palumbo, P., Capria, M. T., et al. 2008, MNRAS, 390, 265 Mazzotta Epifani, E., Dall'Ora, M., Di Fabrizio, L., et al. 2010, A\&A, 513, A33 Mazzotta Epifani E., Dall'Ora M., Perna D. et al. 2011, MNRAS, 415, 3097 Mazzotta Epifani, E., Perna, D., Licandro, J., et al. 2014a, A\&A, 565, A69 Mazzotta Epifani, E., Perna, D., Di Fabrizio, L., et al., 2014b, A\&A, 561, A6 Meech K. J., \& Belton, M. 1989, IAU, Circ., 4770

Meech, K. J., \& Weaver, H. A. 1996, Earth Moon Planets, 72, 119

Meech, K. J., Buie, M. W., Samarasinha, N. H., Mueller, B. E. A., \& Belton, M 1997, AJ, 133, 844

Ortiz J. L., Duffard R., Pinilla-Alonso N. et al. 2015, A\&A 576, A18

Pal, A., Kiss, Cs., Horner, J., et al. 2015, A\&A, 583, A93

Peixinho, N., Boehnhardt, H., Belskaya, I., et al. 2004, Icarus, 170, 153
Perna, D., Dotto, E., Barucci, M. A., et al. 2013, A\&A, 554, A49

Romon-Martin, J., Delahodde, C., Barucci, M. A., de Bergh, C., \& Peixinho, N. 2003 A\&A, 400, 369

Rousselot, P., Petit, J.-M., Poulet, F., \& Sergeev, A. 2005, Icarus, 176, 478

Russell, H. N. 1916, ApJ, 43, 173

Shi, J. C., \& Ma, Y. H. 2015, MNRAS, 454, 3635

Stansberry, J., Grundy, W., Brown, M., et al. 2008, in The Solar System Beyond Neptune, eds. M. A. Barucci, H. Boehnhardt, D. P. Cruikshank, \& A. Morbidelli (The University of Arizona Press)

Tegler, S. C., Bauer, J. M., Romanishin, W., \& Peixinho, N. 2008, in The Solar System Beyond Neptune, eds. M. A. Barucci, H. Boehnhardt, D. P. Cruikshank, \& A. Morbidelli (The University of Arizona Press)

Wainscoat, R., Denneau, J., Hsieh, H., et al. 2011, CBET, 2782, 1 